\title{
Are oral surgeons as good as they think they are?
}

The influence of self-deception and impression management upon self-assessment in oral surgery

\section{A. W. Evans, R. M. A. Leeson, T. R. O. Newton John and A. Petrie. Br Dent J 198: 765-769}

\section{Objective}

To see if poor self-assessment of surgical performance during removal of mandibular third molars is influenced by self-deception (lack of insight) and impression management (trying to convey a favourable impression).

Design

A prospective study of 50 surgeons, surgically removing a lower third molar tooth.

Setting

One UK dental school over a two year period.

Methods

The surgeons' surgical skills were assessed (by two assessors) and self assessed using check-list and global rating scales. Post-operatively, surgeons completed validated deception questionnaires which measured both self-deception enhancement (lack of insight), and impression management (the tendency to deliberately convey a favourable impression).

\section{Main outcome measures}

Reliability between assessors, and between assessors' and surgeons' self-assessments were calculated. Discrepancies between assessors' and surgeons' scores were correlated with surgeons' deception scores.

Results

Reliability between assessors was excellent for checklist (0.96) and global rating scales (0.89) and better than the reliability between assessors and surgeons (0.51 and 0.49). There was a statistically significant correlation $(r=0.45 \mathrm{p}=0.001$ checklist, $r=0.48 \mathrm{p}<0.001$ global) between over/ under-rating of their surgical performance by surgeons and their impression management scores. No statistically significant correlation was found between this inaccuracy in selfassessment and surgeons' individual self-deception scores.

Conclusion

The majority of surgeons scored themselves higher than their assessors for surgical skill in removing a single mandibular third molar tooth. Impression management (the tendency to deliberately convey a favourable impression) may contribute to a surgeon's inaccurate self-reporting of performance. Lack of insight appears to be much less important as a contributing factor. The authors speculate that pressure to provide evidence of good performance may be encouraging surgeons to manage their image and overscore themselves.

\section{IN BRIEF}

- Accurate self-assessment is important for reflection, appraisal and life-long learning.

- Evidence in the literature and this study suggests poor selfassessment is common, both generally in higher education and in medicine and dentistry.

- When surgeons removed a lower third molar tooth, apparent poor self-assessment of performance was more likely to be due to the desire to convey a favourable impression than to lack of insight.

- It is possible that pressure to provide evidence of good performance will encourage surgeons to manage their image and over-score themselves.

\section{COMMENT}

This is an interesting paper examining the influence of selfdeception (defined as a lack of insight) and impression management (the act of conveying a favourable impression) in a group of 50 oral surgeons asked to carry out self-assessment of their operative performance during third molar removal.

The authors observed that the majority of surgeons scored themselves higher than independent assessors for surgical skill, principally due to impression management, and suggest that external pressure to demonstrate good performance may encourage surgeons to present themselves in the most favourable light.

Surgery, of course, is an ancient art and historically the attributes thought necessary to be a successful surgeon included self-confidence, decisiveness and concentration of mind, good eyesight, and sensitive and dextrous hands! ${ }^{1}$ Whilst these descriptions are now amusingly archaic, there must be few surgeons who have not found such attributes invaluable during their operative careers.

Evans et al. rightly raise concerns, however, that surgeons may appear over-confident, arrogant and suffer from lack of insight. What is fascinating, of course, is just where the optimum balance lies between appropriate confidence and decisiveness, necessary for safe and efficient surgery, and an undesirable over-estimate of personal skill.

This paper only deals with one, minor oral surgery procedure but it is a pity that there is no mention of the seniority and training of the surgeons involved. I suspect that a consultant oral and maxillofacial surgeon, for example, will respond differently to assessment than a post-graduate dental student! Nonetheless, objective and reproducible assessments of operative efficiency are here to stay, and as the authors correctly point out, this will affect all levels of surgical training from student assessment and specialist training through to consultant appraisal and revalidation.

It must be remembered, however, that clinical surgery is not merely about good operative technique. It requires the highest standards of patient assessment, diagnostic acumen, appropriate use of imaging and laboratory technique and comprehensive treatment planning, to enable the modern surgeon, in the words of Professor Aird, 'to carry out the correct procedure at the right time.' ${ }^{1}$ Let us not forget this timeless advice as we move towards an era of competency based assessment and measurement of technical proficiency.

\section{P. J. Thomson, Professor of Oral and MaxilloFacial Surgery, University of Newcastle upon Tyne}

1. AIRD I. The Making of a Surgeon. London: Butterworths, 1961.

doi: 10.1038/sj.bdj.4812449 